\title{
MEASURES: CONTINUITY, MEASURABILITY, DUALITY, EXTENSION
}

\author{
ROMAN FRIČ
}

\begin{abstract}
We discuss some basic ideas and survey some fundamental constructions related to measure (a real-valued map the domain of which is a set of measurable objects carrying a suitable structure and the map partially preserves the structure): continuity, measurability, duality, extension. We show that in the category $I D$ of difference posets of fuzzy sets and sequentially continuous difference-homomorphisms these constructions are intrinsic. Further, basic notions of the probability theory have natural generalizations within $I D$.
\end{abstract}

\section{Introduction}

Let $X$ be a nonempty set and let $\mathbb{A}$ be a system of subsets of $X$. Usually, a measure on $\mathbb{A}$ is a map $m$ of $\mathbb{A}$ into nonnegative real numbers $R_{+}$having certain natural properties of a volume. We shall always assume that $\mathbb{A}$ is a ring of subsets and, unless stated otherwise, $m$ is $\sigma$-additive. If $\mathbb{A}$ is a field of sets (i.e., $X \in \mathbb{A}$ ) and $m$ is normed (i.e., $m(X)=1$ ), then $m$ is said to be a probability measure. Having in mind applications to probability theory, our goal is to find a suitable category in which probability measures are morphisms and the usual measuretheoretic constructions can be described in categorical terms. We claim that the category $I D$ of $D$-posets of fuzzy sets (systems of partially ordered fuzzy sets carrying a partial binary operation called difference) and sequentially continuous $D$-homomorphisms $(28],[10])$ is exactly what is needed. We show that $I D$ is a natural category which also covers suitable classes of Boolean algebras, $M V$ -algebras, effect algebras, and also observables, fuzzy random variables, states,

2000 Mathematics Subject Classification: Primary 28-02, 06D72, 18A20; Secondary 06D50, 06D35, 60A05.

Keyw ords: bounded measure, sequential continuity, extension of measures, $D$-poset of fuzzy sets, bold algebra, Łukasiewicz tribe, measurable map, fuzzy random variable, observable, duality, probability measure, state, epireflection, generalized probability.

This work was supported by the Slovak Research and Development Agency under the contract No. APVV-0071-06, Slovak Academy of Sciences via the project Center of Excellence — Physics of Information, and VEGA 2/6088/26. 
etc. Some results can easily be extended to bounded measures. Observe that an unbounded measure $m$ is not sequentially continuous (cf. 25]) and only normed measures are $D$-homomorphisms (cf. [28]).

Primarily, the present paper is intended as a survey, partial results can be found, e.g., in [9], 11], [12], [13], [16], 28], 15]. For the reader's convenience we will recall basic notions as they appear in the text. Detailed information can be found in the references: on category theory in [1], on $M V$-algebras in [4, on $D$-posets and effect algebras in [5], on $I D$ in [10], [28], on fuzzy random variables in 15$]$.

\section{Continuity}

Claim 1. For "nondiscrete" measures and their generalizations (states) sequential continuity is fundamental.

Let $\mathbb{A}$ be a ring of subsets of $X$ and let $m: \mathbb{A} \longrightarrow R_{+}$be a finitely additive measure. It is known that the $\sigma$-additivity of $m$ is equivalent to the additivity and the monotone continuity of $m$ from above: if $A_{n} \searrow \emptyset$, then $m(A) \searrow 0$, where $A_{n} \searrow \emptyset$ means that $\left\langle A_{n}>\right.$ is a nonincreasing sequence in $\mathbb{A}$ and $\emptyset=\bigcap_{n=1}^{\infty} A_{n}$; equivalently, the sequence $\left\langle\chi_{A_{n}}>\right.$ of the characteristic functions $\chi_{A_{n}}$ of $A_{n}$ pointwise (downwards) converges to the constant zero function $\chi_{\emptyset}$. Continuity from below is defined analogously. As usual, we say that a sequence $\left\langle A_{n}\right\rangle$ converges in $\mathbb{A}$ to $A$ if $A=\bigcap_{k=1}^{\infty} \bigcup_{n=k}^{\infty} A_{n}=\bigcup_{k=1}^{\infty} \bigcap_{n=k}^{\infty} A_{n}$ or, equivalently, $<\chi_{A_{n}}>$ converges pointwise to $\chi_{A}$; we write $A=\lim _{n \rightarrow \infty} A_{n}$. It is easy to see that the sequential continuity and the additivity of $m$ implies the $\sigma$-additity of $m$. It is less known that for bounded measures also the converse implication holds, i.e., the $\sigma$-additivity implies the sequential continuity of $m$.

Observe that no unbounded measure is sequentially continuous. Indeed, if a measure $m$ is unbounded, then in $\mathbb{A}$ there exists a sequence $\left\langle B_{n}\right\rangle$ such that the corresponding sequence $<m\left(B_{n}\right)>$ is increasing, say $m\left(B_{n+1}\right)>$ $2 m\left(B_{n}\right), n \in N$, and hence in $\mathbb{A}$ there exists a sequence $\left\langle A_{n}\right\rangle$ of mutually disjoint sets such that $1 \leq m\left(A_{1}\right) \leq m\left(A_{n}\right), n \in N$. Consequently, $\lim _{n \rightarrow \infty} A_{n}=\emptyset$ and $\lim _{n \rightarrow \infty} m\left(A_{n}\right)=0$ does not hold.

Now, let $m$ be a bounded $\sigma$-additive measure on $\mathbb{A}$. 1 . If $\mathbb{A}$ is a $\sigma$-ring, in symbols $\mathbb{A}=\sigma(\mathbb{A})$, then the sequential continuity of $m$ is a simple consequence of the well-known

Lebesgue's Dominated Convergence Theorem. Let $<f_{n}>$ be a sequence of measurable functions converging almost everywhere to $f$. Let $g$ be an integrable function such that $\left|f_{n}\right| \leq g, \quad n \in N, \int g \mathrm{~d} m<+\infty$. Then $f$ is integrable and $\int f \mathrm{~d} m=\lim _{n \rightarrow \infty} \int f_{n} \mathrm{~d} m$. 


\section{MEASURES: CONTINUITY, MEASURABILITY, DUALITY, EXTENSION}

Indeed, for $A=\lim _{n \rightarrow \infty} A_{n}$ it suffices to put $f_{n}=\chi_{A_{n}}, f=\chi_{A}, B=\cup_{n=1}^{\infty} A_{n}$, and $g=\chi_{B}$. However, there is a more simple argument leading to the converse implication (cf. [11]). Assume that $\left\langle B_{n}\right\rangle$ converges in $\mathbb{A}$ to $B$. Put $A_{n}=$ $\cap_{k=n}^{\infty} B_{k}, C_{n}=\cup_{k=n}^{\infty} B_{k}$. Since $\mathbb{A}$ is a $\sigma$-ring, $\left\langle A_{n}\right\rangle$ and $\left\langle C_{n}\right\rangle$ are sequences in $\mathbb{A}$ and it is easy to see that $\left\langle A_{n}\right\rangle$ is nondecreasing, $\left\langle C_{n}\right\rangle$ is nonincreasing, $A_{n} \subseteq B_{n} \subseteq C_{n}$ for all $n \in N, B=\cup_{n=1}^{\infty} A_{n}=\cap_{n=1}^{\infty} C_{n}$. Hence $m\left(A_{n}\right) \leq$ $m\left(B_{n}\right) \leq m\left(C_{n}\right)$ and $m(B)=\lim _{n \rightarrow \infty} m\left(B_{n}\right)$.

If $\mathbb{A} \neq \sigma(\mathbb{A})$, then according to the Measure Extension Theorem (METHM) $m$ can be uniquely extended to a $\sigma$-additive measure on $\sigma(\mathbb{A})$ and the rest is clear.

Virtually, the same argument has been used in [11 to support the claim that for bold algebras (i.e., $M V$-algebras of fuzzy sets) "extendable" nondiscrete measures are sequentially continuous.

Let $I$ be the closed unit interval [0,1] carrying the usual $M V$-algebra operations and order and the usual convergence of sequences:

$$
\begin{aligned}
x \oplus y & =\min \{1, x+y\}, \\
x^{*} & =1-x, \\
x \odot y & =\max \{0, x+y-1\}=\left(x^{*} \oplus y^{*}\right)^{*}, \\
x \vee y & =\max \{x, y\}=\left(x^{*} \oplus y\right)^{*} \oplus y, \\
x \wedge y & =\min \{x, y\}=\left(x^{*} \vee y^{*}\right)^{*}, \\
\lim _{n \rightarrow \infty} x_{n} & =x .
\end{aligned}
$$

Observe that both $\oplus$ and $\odot$ are commutative and associative.

Further, let $X$ be a set and let $I^{X}$ be the set of all functions on $X$ into $I$ carrying the pointwise $M V$-algebra operations and order and the pointwise convergence of sequences, i.e., for each $x \in X$ put:

$$
\begin{aligned}
(f \oplus g)(x) & =f(x) \oplus g(x), \\
f^{*}(x) & =(f(x))^{*}, \\
(f \odot g)(x) & =f(x) \odot g(x), \\
(f \vee g)(x) & =f(x) \vee g(x), \\
(f \wedge g)(x) & =f(x) \wedge g(x), \text { and } \\
\operatorname{Lim}_{n \rightarrow \infty} f_{n} & =f \text { if and only if } \lim _{n \rightarrow \infty} f_{n}(x)=f(x) \text { for each } x \in X .
\end{aligned}
$$

If $\mathcal{A}$ is a subalgebra of $I^{X}\left(\mathcal{A}\right.$ contains the constant functions $1_{X}$ and $0_{X}$ and it is closed with respect to all operations and order and carries the pointwise convergence of sequences), then $\mathcal{A}$ is said to be a bold algebra. A bold algebra $\mathcal{A}$ such that for each sequence $\left\langle f_{n}>\right.$ in $\mathcal{A}$ also $\min \left\{1_{X}, \sum_{n=1}^{\infty} f_{n}\right\}$ belongs to $\mathcal{A}$ is said to be a Eukasiewicz tribe or, simply, a tribe. We shall additionally utilize 
operations $\ominus$ and $\triangle$ defined as follows:

$$
\begin{aligned}
(f \ominus g)(x) & =\max \{0, f(x)-g(x)\}=\left(f \odot g^{*}\right)(x), \\
(f \triangle g)(x) & =\max \{(f \ominus g)(x),(g \ominus f)(x)\} .
\end{aligned}
$$

It is known that each $\sigma$-complete $M V$-algebra is Archimedean (or semisimple) and each Archimedean $M V$-algebra can be represented by a bold algebra (the elements of which are fuzzy subsets of the underlying set of the bold algebra). If for each element $f$ in a bold algebra $\mathcal{A} \subseteq I^{I}$ we have $f(x) \in\{0,1\}, x \in X$, then $\mathcal{A}$ becomes a field of subsets of $X$ (via characteristic functions).

Let $\mathcal{A} \subseteq I^{X}$ be a bold algebra. A map $m: \mathcal{A} \longrightarrow I$ is said to be a measure if it is normed and subtractive, i.e., $m\left(1_{X}\right)=1$ and $m(g \ominus f)=m(g)-m(f)$ whenever $f, g \in \mathcal{A}, f \leq g$ (obviously, $m\left(0_{X}\right)=0$ and $m$ preserves the order). This definition is equivalent to the usual one (also for fields of subsets) and it better suits our purpose.

Lemma 1.1. Let $\mathcal{A} \subseteq I^{X}$ be a bold algebra. Then the following are equivalent:

(i) $\mathcal{A}$ is a Eukasiewicz tribe;

(ii) If $\left\langle f_{n}\right\rangle$ is a nondecreasing (nonincreasing) sequence in $\mathcal{A}$ and $f=\operatorname{Lim}_{n \rightarrow \infty} f_{n}$, then $f \in \mathcal{A}$.

Proof.

(1) (i) implies (ii). The assertion follows from Proposition 8.13 in [33].

(2) (ii) implies (i). Assume (ii) and let $\left\langle g_{n}>\right.$ be a sequence in $\mathcal{A}$. Put $f_{1}=g_{1}$ and, inductively, $f_{n+1}=f_{n} \oplus g_{n+1}, n \geq 1$. Then $<f_{n}>$ is a nondecreasing sequence in $\mathcal{A}$. Since $\min \left\{\sum_{n=1}^{\infty} g_{n}, 1\right\}=\bigvee_{n=1}^{\infty} f_{n}$ (defined pointwise), it follows that $\mathcal{A}$ is a Eukasiewicz tribe.

Lemma 1.2. Let $\mathcal{A} \subseteq I^{X}$ be a Eukasiewicz tribe. Let $\left\langle g_{n}\right\rangle$ be a sequence in $\mathcal{A}$ and let $g \in \mathcal{A}$. Then $\operatorname{Lim}_{n \longrightarrow \infty} g_{n}=g$ if and only if in $\mathcal{A}$ there are sequences $<f_{n}>$ and $<h_{n}>$ such that

$\left(\mathrm{C}_{1}\right)<f_{n}>$ is nondecreasing and $<h_{n}>$ is nonincreasing,

$\left(\mathrm{C}_{2}\right) f_{n} \leq g_{n} \leq h_{n}, n \in N$,

$\left(\mathrm{C}_{3}\right) g=\bigvee_{n=1}^{\infty} f_{n}=\bigwedge_{n=1}^{\infty} h_{n}$.

P r o of. Necessity. Assume that $\left\langle g_{n}>\right.$ converges to $g$ in $\mathcal{A}$. Put $f_{n}=\bigwedge_{k=n}^{\infty} g_{k}$, $h_{n}=\bigvee_{k=n}^{\infty} g_{k}$ (defined pointwise). Since $\mathcal{A}$ is closed with respect to monotone limits, $\left\langle f_{n}>\right.$ and $\left\langle h_{n}>\right.$ are sequences in $\mathcal{A}$ and it is easy to see that conditions $\left(\mathrm{C}_{1}\right),\left(\mathrm{C}_{2}\right),\left(\mathrm{C}_{3}\right)$ are satisfied. 


\section{MEASURES: CONTINUITY, MEASURABILITY, DUALITY, EXTENSION}

Sufficiency. Assume that $\left(\mathrm{C}_{1}\right),\left(\mathrm{C}_{2}\right),\left(\mathrm{C}_{3}\right)$ hold true. It is easy to verify that $\lim _{n \rightarrow \infty} g_{n}(x)=g(x)$ for each $x \in X$. Hence $\operatorname{Lim}_{n \rightarrow \infty} g_{n}=g$ and the proof is complete.

Corollary 1.3. Let $\mathcal{A} \subseteq I^{X}$ be a Eukasiewicz tribe. Then the pointwise convergence $\operatorname{Lim}$ in $\mathcal{A}$ is the finest of all sequential convergences $\mathbb{K}$ in $\mathcal{A}$ such that each nondecreasing sequence $<f_{n}>$ converges under $\mathbb{K}$ to $\bigvee_{n=1}^{\infty} f_{n}$, each nonincreasing sequence $<h_{n}>$ converges under $\mathbb{K}$ to $\bigvee_{n=1}^{\infty} h_{n}$ and a sequence $\left\langle g_{n}>\right.$ converges under $\mathbb{K}$ to $g$ whenever in $\mathcal{A}$ there are sequences $\left\langle f_{n}\right\rangle$ and $\left\langle h_{n}\right\rangle$ satisfying conditions $\left(\mathrm{C}_{1}\right),\left(\mathrm{C}_{2}\right),\left(\mathrm{C}_{3}\right)$.

Corollary 1.4. Let $\mathcal{A} \subseteq I^{X}$ be a bold algebra. Then $\mathcal{A}$ is a Lukasiewicz tribe if and only if $\mathcal{A}$ is a sequentially closed subset of $I^{X}$ (with respect to the pointwise convergence).

Let $\mathcal{A} \subseteq I^{X}$ be a bold algebra. Then the smallest sequentially closed subset of $I^{X}$ containing $\mathcal{A}$ is a Eukasiewicz tribe; it is called the generated Eukasiewicz tribe and it is denoted by $\sigma(\mathcal{A})$. Symbol $f_{n} \nearrow f$ means that $\left\langle f_{n}\right\rangle$ is a nondecreasing sequence and $f=\bigvee_{n=1}^{\infty} f_{n} ; f_{n} \searrow f$ is defined analogously. Let $m$ be a measure on $\mathcal{A}$. If $f_{n} \nearrow f$ and $f \in \mathcal{A}$ implies $m\left(f_{n}\right) \nearrow m(f)$, then $m$ is said to be sequentially continuous from below; sequential continuity from above is defined analogously.

Corollary 1.5. Let $\mathcal{A} \subseteq I^{X}$ be a Eukasiewicz tribe and let $m: \mathcal{A} \longrightarrow I$ be a measure sequentially continuous from below (equivalently, sequentially continuous from above). Then $m$ is sequentially continuous.

P r o of. 1. Let $\mathcal{A} \subseteq I^{X}$ be a Eukasiewicz tribe. Let $\operatorname{Lim}_{n \longrightarrow \infty} g_{n}=g$ in $\mathcal{A}$. According to Lemma 2.2 , in $\mathcal{A}$ there are sequences $\left\langle f_{n}>\right.$ and $<h_{n}>$ satisfying conditions $\left(\mathrm{C}_{1}\right),\left(\mathrm{C}_{2}\right),\left(\mathrm{C}_{3}\right)$. Clearly, $f_{n} \leq g_{n} \leq h_{n}$ implies $m\left(f_{n}\right) \leq m\left(g_{n}\right) \leq$ $m\left(h_{n}\right), n \in N$. Since $m$ is sequentially continuous both from below and from above, $\lim _{n \rightarrow \infty} m\left(f_{n}\right)=\lim _{n \rightarrow \infty} m\left(h_{n}\right)=m(g)$ implies $\lim _{n \rightarrow \infty} m\left(g_{n}\right)=m(g)$.

In 11 the following variant of the METHM has been proved.

TheOrem 1.6. Let $\mathcal{A} \subseteq I^{X}$ be a bold algebra, let $\sigma(\mathcal{A}) \subseteq I^{X}$ be the generated Eukasiewicz tribe, and let $m: \mathcal{A} \longrightarrow I$ be a sequentially continuous measure. Then $m$ can be extended to a sequentially continuous measure $m_{\sigma}: \sigma(\mathcal{A}) \longrightarrow I$ and the extension is uniquely determined.

TheOrem 1.7. Let $\mathcal{A} \subseteq I^{X}$ be a bold algebra and let $m: \mathcal{A} \longrightarrow I$ be a measure sequentially continuous from below (equivalently, sequentially continuous from above). Let $\sigma(\mathcal{A}) \subseteq I^{X}$ be the generated Eukasiewicz tribe. If $m$ can be extended to a measure $m_{\sigma}: \sigma(\mathcal{A}) \rightarrow I$ which is sequentially continuous from below, then $m$ is sequentially continuous. 
P r o of. Assume that $m$ can be extended to a measure $m_{\sigma}: \sigma(\mathcal{A}) \longrightarrow I$ sequentially continuous from below (cf. [20]). According to Corollary 2.5, $m_{\sigma}$ is sequentially continuous, hence its restriction $m$ is sequentially continous on $\mathcal{A}$.

Corollary 1.8. Let $\mathcal{A}$ be a bold algebra and let $m$ be a measure on $\mathcal{A}$. Let $\sigma(\mathcal{A})$ be the generated Lukasiewicz tribe Then the following are equivalent:

(i) $m$ is sequentially continuous;

(ii) $m$ is sequentially continuous from below and it can be extended over $\sigma(\mathcal{A})$ to a measure sequentially continuous from below;

(iii) $m$ is sequentially continuous from above and it can be extended over $\sigma(\mathcal{A})$ to a measure sequentially continuous from above.

Finally, in 25] there is a direct proof of the fact that the $\sigma$-additivity of a bounded measure on a ring of subsets implies its sequential continuity. In [9] the proof has been generalized to Boolean algebras carrying a suitable sequential convergence (initial with respect to a separating system of Boolean homomorphisms into the two-point Boolean algebra; it is of the same nature as the pointwise convergence for fields of subsets).

\section{Measurability}

Claim 2. The measurability of a map can be formulated in a categorical way (via composition of maps).

Let $X$ be a set and let $\mathbb{A}$ be a system of subsets of $X$. Then $(X, \mathbb{A})$ is said to be a measurable space. Let $(Y, \mathbb{B})$ be another measurable space and let $f$ be a map of $X$ into $Y$ such that for each $B \in \mathbb{B}$ the preimage $f^{\triangleleft}(B)=\{x \in X ; f(x) \in B\}$ belongs to $\mathbb{A}$, then $f$ is said to be $(\mathbb{A}, \mathbb{B})$-measurable or, simply, measurable. If $\mathbb{A}$ and $\mathbb{B}$ are fields of sets, then the induced preimage map $f^{\triangleleft}$ of $\mathbb{B}$ into $\mathbb{A}$ is a Boolean homomorphism and it is called an observable. It induces a map $T_{f}$ of the set $\mathcal{P}(\mathbb{A})$ of all probability measures on $\mathbb{A}$ into the set $\mathcal{P}(\mathbb{B})$ of all probability measures on $\mathbb{B}$ defined by $\left(T_{f}(p)\right)(B)=p\left(f^{\triangleleft}(B)\right), B \in \mathbb{B}, p \in \mathcal{P}(\mathbb{A})$; it is called the distribution of $f$ and it can be viewed as a channel transforming probability information from a probability space $(X, \mathbb{A}, p)$ into the probability space $\left(Y, \mathbb{B}, T_{f}(p)\right)$. If $Y$ is the real line $R$ and $\mathbb{B}$ is the set of all Borel measurable sets, then $f$ is said to be a random variable. In [8] the following theorem has been proved.

Theorem 2.1. The preimage map $f^{\triangleleft}$ is a sequentially continuous Boolean homomorphism of $\mathbb{B}$ into $\mathbb{A}$. 


\section{MEASURES: CONTINUITY, MEASURABILITY, DUALITY, EXTENSION}

The proof is based on a simple observation that for each map $f: X \longrightarrow Y$ and for each subset $B$ of $Y$ the composition $\chi_{B} \circ f$ is the characteristic function $\chi_{f} \triangleleft(B)$ of $f^{\triangleleft}(B)$. Hence the measurability of $f$ can be restated via the composition: $f$ is measurable if and only if for each $B \in \mathbb{B}$ the composition $\chi_{B} \circ f$ belongs to $\mathbb{A}$.

In fact, Theorem 2.1. characterizes a measurable map and it leads ([10]) to an abstract (categorical) definition: $f$ is said to be measurable if $f^{\triangleleft}$ is a morphism (sequentially continuous Boolean homomorphism). In other words, $\mathbb{A}$ can be considered as the initial Boolean structure on $X$ defined by a cone of arrows $\left\{\chi_{A}: X \longrightarrow\{0,1\} ; A \in \mathbb{A}\right\}$, similarly $\mathbb{B}$ can be considered as the initial Boolean structure on $Y$ defined by a cone of arrows $\left\{\chi_{B}: X \longrightarrow\{0,1\}\right.$; $B \in \mathbb{B}\}$, and the measurability of $f$ means that the cone of composed arrows $\left\{\chi_{A} \circ f: Y \longrightarrow\{0,1\} ; A \in \mathbb{A}\right\}$ is contained in $\left\{\chi_{B}: X \longrightarrow\{0,1\} ; B \in \mathbb{B}\right\} ;$ this means that $f^{\triangleleft}: \mathbb{B} \longrightarrow \mathbb{A}$ is a morphism.

Fuzzy (also operational) random variables generalize the classical case in two directions: fuzzy and quantum. As usually, fuzzy means that the membership function has values from the interval $[0,1]$. The quantum aspect is, unlike in the classical case where a random variable $f$ maps the elementary events (points, i.e., point-measures) into the elementary events (and via $T_{f}, \mathcal{P}(\mathbb{A})$ into $\mathcal{P}(\mathbb{B})$ ), a fuzzy random variable maps the elementary events into the probability measures and the image of a point can be a nondegenerated probability measure, hence the corresponding observable (as a map of subsets to subsets) sends crisp subsets into fuzzy subsets. The interested reader can find more information about fuzzy random variables in [2], 3], [19], [15, [29]. Here we restrict ourselves to some basic facts.

Definition 2.2. Let $\mathbb{A}$ and $\mathbb{B}$ be $\sigma$-fields of subsets of $\Omega$ and $\Xi$, respectively and let $(\Omega, \mathbb{A}),(\Xi, \mathbb{B})$ be measurable spaces. Let $T$ be a map of $P(\mathbb{A})$ into $P(\mathbb{B})$ such that, for each $B \in \mathbb{B}$, the assignment $\omega \mapsto\left(T\left(\delta_{\omega}\right)\right)(B)$ yields a measurable map of $\Omega$ into $[0,1]$ and

$$
(T(m))(B)=\int\left(T\left(\delta_{\omega}\right)\right)(B) \mathrm{d} m
$$

for all $m \in P(\mathbb{B})$ and all $B \in \mathbb{B}$. Then $T$ is said to be a fuzzy random variable (in the sense of Bugajski and Gudder).

Observe that if $f$ is a classical measurable map of $\Omega$ into $\Xi$, then the distribution $T_{f}$ is a fuzzy random variable. Indeed, $\left(T_{f}\left(\delta_{\omega}\right)\right)(B)=1$ if and only if $f(\omega) \in B$ and $(\mathrm{BG})$ means $T_{f}(m)=m \circ f^{\triangleleft}$.

Denote $\mathcal{M}(\mathbb{A})$ the set of all measurable functions of $\Omega$ into $[0,1]$ and for $u \in \mathcal{M}(\mathbb{A})$, define a map $e v(u): P(\mathbb{A}) \longrightarrow[0,1]$ by $(e v(u))(p)=\int u(\omega) \mathrm{d} p$, $p \in \mathcal{P}(\mathbb{A})$. Put $\mathcal{E}(\mathbb{A})=\{e v(u) ; u \in \mathcal{M}(\mathbb{A})\}$. 
Definition 2.3. A map $T: \mathcal{P}(\mathbb{A}) \longrightarrow \mathcal{P}(\mathbb{B})$ is said to be measurable if for each $u \in \mathcal{M}(\mathbb{B})$ there exists $v \in \mathcal{M}(\mathbb{A})$ such that $e v(u) \circ T=e v(v)$, i.e., $\mathcal{E}(\mathbb{B}) \circ T$ $\subseteq \mathcal{E}(\mathbb{A})$.

TheOREM 2.4. Let $T$ be a map of $\mathcal{P}(\mathbb{A})$ into $\mathcal{P}(\mathbb{B})$. Then the following are equivalent:

(i) $T$ is a fuzzy random variable;

(ii) $T$ is measurable.

Observe that a measurable map $T$ of $\mathcal{P}(\mathbb{A})$ into $\mathcal{P}(\mathbb{B})$ induces a sequentially continuous map $T^{\triangleleft}$ of $\mathcal{E}(\mathbb{B})$ into $\mathcal{E}(\mathbb{A})$. In the next section it will be shown that the sets $\mathcal{E}$ carry a natural $I D$ structure and $T^{\triangleleft}$ is a morphism (observable).

\section{Duality}

Claim 3. D-posets of fuzzy sets are exactly what is needed.

In this section we outline a (nontopological) Stone-type duality suitable for applications in probability.

Let $f$ be a measurable map of a measurable space $(X, \mathbb{A})$ into a measurable space $(Y, \mathbb{B})$. Then $f^{\triangleleft}$ is a Boolean homomorphism of $\mathbb{B}$ into $\mathbb{A}$. The problem is whether each Boolean homomorphism $h$ of $\mathbb{B}$ into $\mathbb{A}$ can be represented as $g^{\triangleleft}$ for some measurable map $g$ of $X$ into $Y$. The famous Stone duality (the nontopological variant) gives the answer. If $\mathbb{A}$ and $\mathbb{B}$ are reduced (i.e., for each pair of points, the first belongs to a measurable set and the other belongs into its complement) and perfect (i.e., each ultrafilter of measurable sets is fixed), then for each $h$ there exists a unique $g$ such that $h=g^{\triangleleft}$. Even though the field of real Borel sets is not perfect, some other argument guarantees that the random variables and observables are in a one-to-one correspondence. Indeed, perfectness has to be replaced with $s$-perfectness (each ultrafilter of measurable sets having the countable intersection property is fixed), cf. [8]. Observe that $f^{\triangleleft}$ and $T^{\triangleleft}$ are sequentially continuous (see Section 3), hence it is natural to restrict our attention to sequentially continuous "observables". We aim at a duality covering both the fields of sets and their fuzzy and quantum generalizations.

Recall (cf. [23]) that a $D$-poset is a quintuple $\left(X, \leq, \ominus, 0_{X}, 1_{X}\right)$ where $X$ is a set, $\leq$ is a partial order, $0_{X}$ is the least element, $1_{X}$ is the greatest element, $\ominus$ is a partial operation on $X$, called difference, such that $a \ominus b$ is defined if and only if $b \leq a$, and the following axioms are assumed:

(D1) $a \ominus 0_{X}=a$ for each $a \in X$;

(D2) If $c \leq b \leq a$, then $a \ominus b \leq a \ominus c$ and $(a \ominus c) \ominus(a \ominus b)=b \ominus c$. 
If no confusion can arise, then the quintuple $\left(X, \leq, \ominus, 0_{X}, 1_{X}\right)$ is condensed to $X$. A map $h$ of a $D$-poset $X$ into a $D$-poset $Y$ which preserves the $D$-structure is said to be a D-homomorphism.

ExAmple 3.1. Let $(X, \mathbb{A})$ be a measurable space. Define a partial operation " $\ominus$ " as follows: for $A, B \in \mathbb{A}, B \subseteq A$, put $A \ominus B=A \backslash B$. Then $\mathbb{A}$, carrying the partial order by inclusion, together with the difference is an example of a $D$-poset. If $\mathbb{A}$ is viewed as the set of all corresponding characteristic functions carrying the pointwise convergence, then $\mathbb{A}$ becomes a $D$-poset of crisp sets ( $\{0,1\}$-valued, the order and the difference are defined pointwise).

ExAMPLE 3.2. Consider the unit interval $I=[0,1]$ carrying the natural order, algebraic operations and convergence. Define a partial operation " $\ominus$ " as follows: for $a, b \in I, b \leq a$, put $a \ominus b=a-b$. Then $I$ carrying the natural (total) order, together with the partial operation is a $D$-poset. Let $X$ be a set. Consider $I^{X}$ carrying the pointwise partial order and the difference defined pointwise: for $f, g \in I^{X}, g \leq f$ (i.e., $g(x) \leq f(x)$ for all $\left.x \in X\right)$, put $(f \ominus g)(x)=f(x)-g(x)$, $x \in X$. If $X$ is a singleton, then $I$ and $I^{X}$ are isomorphic. Assume that $\mathcal{X} \subseteq I^{X}$ contains the constant fuctions "zero" and "one" and it is closed with respect to the pointwise difference. Then $\mathcal{X}$ is a typical $D$-poset we are interested in; it will be called a $D$-poset of fuzzy sets (see [22]). Fields of sets and bold algebras can be viewed as special cases. Indeed, the difference $\chi_{A} \ominus \chi_{B}$, resp. $f \ominus g$, is defined only for $B \subseteq A$, resp. $g \leq f$, and then $\chi_{A} \ominus \chi_{B}=\chi_{A \backslash B}$, resp. $f \ominus g=f-g$. If $\mathcal{X} \subseteq I^{X}$ is a a bold algebra closed with respect to the pointwise limits, then it is a Lukasiewicz tribe. Each sequentially continuous measure on a bold algebra is a sequentially continuous $D$-homomorphism. Each $x \in \mathcal{X}$ can be considered as a sequentially continuous $D$-homomorphism of $\mathcal{X}$ into $I$, hence a sequentially continuous measure; it is called the evaluation at $x$ and denoted by $e v_{x}$.

Definition 3.3. Let $\mathcal{X} \subseteq I^{X}$ be a $D$-poset of fuzzy sets. If for each pair $x, y \in X, x \neq y$, there exists $u \in \mathcal{X}$ such that $u(x) \neq u(y)$, then $\mathcal{X}$ is said to be reduced. A morphism of $\mathcal{X}$ into $I$ is said to be a state. If for each state $s$ there exists a unique $x \in \mathcal{X}$ such that $s=e v_{x}$, then $\mathcal{X}$ is said to be sober.

Define the following category $I D$ : the objects are reduced $D$-posets of fuzzy sets carrying the pointwise sequential convergence, the morphisms are sequentially continuous $D$-homomorphisms. More information about $I D$ and its properties can be found in [13, [28]. Denote $F S$ the full subcategory of $I D$ the objects of which are fields of sets and the morphisms are sequentially continuous $D$-homomorphisms. In what follows we assume that all $D$-posets of fuzzy sets are reduced.

Lemмa 3.4. Let $\mathbb{A}$ be a field of subsets of $X$, let $\mathbb{B}$ be a field of subsets of $Y$, let $h$ be a map of $\mathbb{A}$ into $\mathbb{B}$, and let $p$ be a map of $\mathbb{A}$ into $I$. 
(i) Let $h$ be a D-homomorphism. Then $h$ is a Boolean homomorphism.

(ii) Let $p$ be a D-homomorphism. Then $p$ is an additive normed measure.

Pr o of. A straightforward calculation (cf. [28, Lemma 3.3]) is omitted.

Observe that if $p$ is a probability measure on a field of subsets $\mathbb{A}$, then $p$ can be considered as a sequentially continuous $D$-homomorphism of $\mathbb{A}$ into $I$. In fact, confirming Claim 3, the sequentially continuous $D$-homomorphisms of $\mathbb{A}$ into $I$ correspond exactly to probability measures on $\mathbb{A}$.

CoRollary 3.5. The category of reduced fields of sets and sequentially continuous Boolean homomorphisms and the category FS are isomorphic. The morphisms of FS ranging in $I$ are exactly the probability measures on fields of sets.

Let $f$ be a map of a nonempty set $X$ into a nonempty set $Y$. Then $f$ induces a map $F$ of $I^{Y}$ into $I^{X}$ defined as follows: $F(u)=u \circ f, u \in I^{Y}$. It is easy to see that $F$ is a sequentially continuous (with respect to the pointwise sequential convergence) $D$-homomorphism. Let $\mathcal{X} \subseteq I^{X}$ and $\mathcal{Y} \subseteq I^{Y}$ be $D$-posets of fuzzy sets such that for each $u \in \mathcal{Y}$ we have $u \circ f \in \mathcal{X}$. Then $F$ restricted to $\mathcal{Y}$ is an $I D$-morphism of $\mathcal{Y}$ into $\mathcal{X}$. Denote it $f^{\triangleleft}$.

Definition 3.6. Let $\mathcal{X} \subseteq I^{X}, \mathcal{Y} \subseteq I^{Y}$ be $D$-posets of fuzzy sets and let $f$ be a map of $X$ into $Y$ such that $f^{\triangleleft}$ is an $I D$-morphism of $\mathcal{Y}$ into $\mathcal{X}$. Then $f$ is said to be $(\mathcal{Y}, \mathcal{X})$-measurable or, simply, measurable.

Denote $M I D$ the following category: objects are pairs $(X, \mathcal{X})$, where $\mathcal{X} \subseteq I^{X}$ is a $D$-poset of fuzzy sets and morphisms are measurable maps. If $\mathcal{X}$ has some property, then we say that $(X, \mathcal{X})$ has the property in question, too.

A natural question arises whether each $I D$-morphism $h$ of $\mathcal{Y}$ into $\mathcal{X}$ is of the form $g^{\triangleleft}$ for some $g: X \longrightarrow Y$. A complete answer is provided by a general categorical duality described in [10] and its specification in [28].

Theorem 3.7. Let $(X, \mathcal{X})$ and $(Y, \mathcal{Y})$ be measurable spaces and let $h$ be a sequentially continuous D-homomorphism of $\mathcal{Y}$ into $\mathcal{X}$. If $\mathcal{Y}$ is sober, then there is a unique measurable map $f$ of $X$ into $Y$ such that $h=f^{\triangleleft}$.

CoRollary 3.8. The subcategory SID of sober objects of ID and the subcategory SMID of sober objects of MID are dually isomorphic.

COROLlaRY 3.9. The category ID and the subcategory SMID of sober objects of $M I D$ are dually naturally equivalent.

Observe that the sobriety of $I D$-objects is a necessary condition for the duality. More general duality between $D$-posets admitting order determining systems of states and measurable spaces has been constructed in [13], see also [8], [12]. 


\section{MEASURES: CONTINUITY, MEASURABILITY, DUALITY, EXTENSION}

In probability theory, the duality between (generalized) random variables (i.e., measure preserving measurable maps) and (generalized) observables yields a bridge between the pointless (algebraic) approach to random events and random functions and the more traditional models based on elementary events (points of the sample probability spaces).

\section{Extension}

Claim 4. Extensions of measures are categorical constructions.

Let $\mathbb{A}$ be a field of subsets of $X$ and let $\sigma(\mathbb{A})$ be the generated $\sigma$-field of subsets of $X$. It is known (cf. [25], 26, [27]) that $\sigma(\mathbb{A})$ is the smallest of all sequentially closed fields $\mathbb{B}$ of subsets of $X$ such that $\mathbb{A} \subseteq \mathbb{B}$; indeed $\sigma(\mathbb{A})$ is the intersection of all such $\mathbb{B}$. Anologously (see [28]), for each $D$-poset $\mathcal{X}$ of fuzzy subsets of $X$ there exists the smallest of all sequentially closed $D$-posets of fuzzy subsets of $X$ containing $\mathcal{X}$; denote it $\sigma(\mathcal{X})$. If $\mathcal{X}$ is a bold algebra, then $\sigma(\mathcal{X})$ can be described via iterated sequential closures.

For $\mathcal{Y} \subseteq I^{X}$, let $\operatorname{cl} \mathcal{Y}$ be the set of all $f \in I^{X}$ such that in $\mathcal{Y}$ there exists a sequence $\left\langle f_{n}>\right.$ converging to $f$ (in the pointwise sequential convergence). For each ordinal number $\alpha$ define $\mathrm{cl}^{\alpha} \mathcal{Y}$ as follows: $\mathrm{cl}^{0} \mathcal{Y}=\mathcal{Y}, \mathrm{cl}^{\alpha} \mathcal{Y}=\mathrm{cl}\left(\mathrm{cl}^{\alpha-1} \mathcal{Y}\right)$ if $\alpha$ is an isolated ordinal number and $\operatorname{cl}^{\alpha} \mathcal{Y}=\operatorname{cl}\left(\bigcup_{\beta<\alpha} \mathrm{cl}^{\beta} \mathcal{Y}\right)$ if $\alpha$ is a limit ordinal number. It is known (cf. [26]) that each $\mathrm{cl}^{\alpha}$ is a closure operator, $\operatorname{cl}\left(\mathrm{cl}^{\omega_{1}} \mathcal{Y}\right)=\mathrm{cl}^{\omega_{1}} \mathcal{Y}$, $\mathrm{cl}^{\omega_{1}}$ is a topological (idempotent) closure operator, and $\mathrm{cl}^{\omega_{1}} \mathcal{Y}$ is the smallest sequentially closed subset of $I^{X}$ which contains $\mathcal{Y}$.

Lemma 4.1. Let $\mathbb{A}$ be a field of subsets of $X$ and let $\mathcal{X}$ be a D-poset of fuzzy subsets of $X$.

(i) $\operatorname{cl}^{\omega_{1}} \mathbb{A}=\sigma(\mathbb{A})$;

(ii) If $\mathcal{X}$ is a bold algebra, then $\mathrm{cl}^{\omega_{1}} \mathcal{X}=\sigma(\mathcal{X})$;

(iii) If $\mathcal{X}$ is a field of (crisp) subsets of $X$, then $\sigma(\mathcal{X})$ is the generated $\sigma$-field.

Proof.

(i) It is easy to see that if $\mathbb{B}$ is a field of subsets of $X$, then $\mathrm{cl} \mathbb{B}$ is a field of subsets of $X$, too. Since the union of a chain of fields of subsets of $X$ is a field of subsets of $X$, it follows that $\mathrm{cl}^{\omega_{1}} \mathbb{A}$ is the smallest of all sequentially closed fields of subsets of $X$ containing $\mathbb{A}$. Hence $\mathrm{cl}^{\omega_{1}} \mathbb{A}=\sigma(\mathbb{A})$.

(ii) The proof is analogous as in the previous case and it is omitted.

(iii) Crisp sets are $\{0,1\}$-valued and $\{0,1\}$-valued bold algebras are exactly fields of sets. Now, the assertion follows from (ii).

This completes the proof. 
Definition 4.2. Let $\mathcal{X}$ be an object of $I D$. If for each morphism $h$ of $\mathcal{X}$ into $I$ there exists a morphism $\bar{h}$ of $\sigma(\mathcal{X})$ into $I$ such that $\bar{h}(u)=h(u)$ for all $u \in \mathcal{X}$, then $\mathcal{X}$ is said to be sufficient.

Recall (cf. [28, Lemma 2.7]), that if $h$ and $h^{\prime}$ are two morphisms of $\sigma(\mathcal{X})$ into an object $\mathcal{Y}$ of $I D$ such that $h(u)=h^{\prime}(u)$ for all $u \in \mathcal{X}$, then $h=h^{\prime}$. Consequently, $\bar{h}$ in the definition above is determined uniquely.

Denote $S T I D$ the full subcategory of $I D$ consisting of sufficient objects. Denote $C I D$ the full subcategory of $I D$ consisting of sequentially closed objects. Clearly, each sequentially closed object of $I D$ is sufficient. Hence $C I D$ is a full subcategory of $S T I D$. Let $\mathbb{A}$ be a field of sets. Since probability measures on $\mathbb{A}$ are exactly sequentially continuous $D$-homomorphisms of $\mathbb{A}$ into $I$ and probability measures on $\mathbb{A}$ can be uniquely extended to probability measures on $\sigma(\mathbb{A})$, it follows that $\mathbb{A}$ as an object of $F S$ is sufficient. The next general extension theorem ([16, Theorem 3.7]) supports our Claim 4.

TheOREM 4.3. CID is an epireflective subcategory of STID.

Proof. The proof is based on the categorical properties of a product. Let $\mathcal{X} \subseteq I^{X}$ be an object of $S T I D$. We claim that the embedding of $\mathcal{X}$ into $\sigma(\mathcal{X})$ is the desired epireflection (remember, $\sigma(\mathcal{X})$ is sequentially closed), i.e., each morphism of $\mathcal{X}$ into a sequentially closed object $\mathcal{Y}$ of $I D$ can be extended to a unique morphism over $\sigma(\mathcal{X})$ to $\mathcal{Y}$ and the embedding of $\mathcal{X}$ into $\sigma(\mathcal{X})$ is an epimorphism (two morphisms of $\sigma(\mathcal{X})$ to the same target object in $I D$ agreeing on $\mathcal{X}$ are identical).

(i) Let $h$ be a morphism of $\mathcal{X}$ into $I$. Since $\mathcal{X}$ is sufficient, it follows that $h$ can be uniquely extended to a morphism $\bar{h}$ of $\sigma(\mathcal{X})$ into $I$.

(ii) Let $\mathcal{Y} \subseteq I^{Y}$ be an object of $C I D$. Let $h$ be a morphism of $\mathcal{X}$ into $\mathcal{Y}$. Since $I^{Y}$ is the categorical product in $I D$, the composition of $h$ (considered as a morphism of $\mathcal{X}$ into $I^{Y}$ ) and each projection of $I^{Y}$ into a factor $I$ is a morphism of $\mathcal{X}$ into $I$ and, according to (i), it can be uniquely extended to a morphism of $\sigma(\mathcal{X})$ to $I$. From the definition of a product and from [28, Lemma 2.7] it follows that there exists a unique morphism $\bar{h}$ of $\sigma(\mathcal{X})$ into $\sigma(\mathcal{Y})=\mathcal{Y}$ such that $\bar{h}(u)=h(u)$ for all $u \in \mathcal{X}$.

This completes the proof.

\section{Corollary 4.4.}

(i) Let $\mathbb{A}$ be a field of sets and let $\sigma(\mathbb{A})$ be the generated $\sigma$-field. Then $\sigma(\mathbb{A})$ is the epireflection of $\mathbb{A}$ as an object of STID into CID.

(ii) Let $\mathcal{X}$ be a bold algebra and let $\sigma(\mathcal{X})$ be the generated Eukasiewicz tribe. Then $\sigma(\mathcal{X})$ is the epireflection of $\mathcal{X}$ as an object of STID into CID. 


\section{Concluding remarks}

Claim 5. The category ID is a natural base category for generalized probability.

Let $(\Omega, \mathbb{A}, p)$ be a probability space in the classical Kolmogorov sense. A measurable map $f$ of $\Omega$ into the real line $R$, called random variable, sends $p$ into a probability masure $p_{f}$, called the distribution of $f$, on the real Borel sets $\mathbb{B}$ via $p_{f}(B)=p\left(f^{\triangleleft}(B)\right), B \in \mathbb{B}$. In fact, $f$ induces a map sending probability measures on $\mathbb{A}$ into probability measures on $\mathbb{B}$ (each point $\omega \in \Omega$, or $r \in R$ is considered as a degenerated point probability measure). The preimage map $f^{\triangleleft}$, called observable, maps $\mathbb{B}$ into $\mathbb{A}$ and it is a sequentially continuous Boolean homomorphism. A fuzzy random variable (or operational r. v.) is a "measurable" map sending probability measures on one probability space into probability measures on another probability space, but it can happen that a point $\omega \in \Omega$ is mapped to a nondegenerated probability measure. The corresponding observable is still sequentially continuous, but sends fuzzy subsets into fuzzy subsets (the image of a crisp set need not be crisp) and preserves some operations on fuzzy sets.

As shown in the previous sections, observables are morphisms in $I D$ and, what is more important, probability measures become morphisms in ID.

Details about fuzzy probability theory can be found, e.g., in [19], [2], [3, [13], [15], 29]. Note that many technical constructions in [2] and [3] are straightforward and transparent (see [15], 29]) in $I D$.

\section{REFERENCES}

[1] ADÁMEK, J.: Theory of Mathematical Structures. Reidel, Dordrecht, 1983.

[2] BUGAJSKI, S.: Statistical maps I. Basic properties, Math. Slovaca 51 (2001), 321-342.

[3] BUGAJSKI, S.: Statistical maps II. Operational random variables, Math. Slovaca 51 (2001), 343-361.

[4] Cignoli, R.-D'Ottaviano, I. M. L.-MUNDiCI, D.: Algebraic Foundations of Many-Valued Reasoning. Kluwer Academic Publ., Dordrecht, 2000.

[5] DVUREČEnSKIJ, A.-PULMANNOVÁ, S.: New Trends in Quantum Structures. Math. Appl., Vol. 516, Kluwer Academic Publ., Dordrecht; Ister Science, Bratislava, 2000.

[6] FOULIS, D. J.-BENNETT, M. K.: Effect algebras and unsharp quantum logics, Found. Phys. 24 (1994), 1331-1352.

[7] FRIČ, R.: Remarks on sequential envelopes, Rend. Istit. Math. Univ. Trieste 20 (1988), 19-28.

[8] FRIČ, R.: A Stone type duality and its applications to probability, Topology Proc. 22 (1999), 125-137.

[9] FRIČ, R.: Boolean algebras: convergence and measure, Topology Appl. 111 (2001), 139-149.

[10] FRIČ, R.: Convergence and duality, Appl. Categ. Structures 10 (2002), 257-266.

[11] FRIČ, R.: Eukasiewicz tribes are absolutely sequentially closed bold algebras, Czechoslovak Math. J. 52 (2002), 861-874. 


\section{ROMAN FRIČ}

[12] FRIČ, R.: Measures on MV-algebras, Soft Comput. 7 (2002), 130-137.

[13] FRIČ, R.: Duality for generalized events, Math. Slovaca 54 (2004), 49-60.

[14] FRIČ, R.: Coproducts of D-posets and their applications to probability, Internt. J. Theoret. Phys. 43 (2004), 1625-1632.

[15] FRIČ, R.: Remarks on statistical maps and fuzzy (operational) random variables, Tatra Mt. Math. Publ. 30 (2005), 21-34.

[16] FRIČ, R.: Extension of measures: a categorical approach, Math. Bohemica 130 (2005), 397-407.

[17] FRIČ, R.-JAKUBÍK, J.: Sequential convergences on Boolean algebras defined by systems of maximal filters, Czechoslovak Math. J. 51 (2001) 261-274.

[18] FRIČ, R.-MCKENNON, K.-RICHARDSON, G. D.: Sequential convergence in $C(X)$, in: Proc. Internat. Summer School-Convergence Structures and Applications to Analysis, Frankfurt a.d. Oder, 1978, Abh. Akad. Wiss. DDR, Abt. Math.-Natur.-Technik, Vol. 4, 1979, Akademie-Verlag, Berlin, 1980, pp. 57-65.

[19] GUDDER, S.: Fuzzy probability theory, Demonstratio Math. 31 (1998), 235-254.

[20] JUREČKOVÁ, M.: The measure extension theorem for MV-algebras, Tatra Mt. Math. Publ. 6 (1995), 56-61.

[21] KENT, D. C.-RICHARDSON, G. D.: Two generalizations of Novák's sequential envelope, Math. Nachr. 19 (1979), 77-85.

[22] KÔPKA, F.: D-posets of fuzzy sets, Tatra Mt. Math. Publ. 1 (1992), 95-102.

[23] KÔPKA, F.-CHOVANEC, F.: D-posets, Math. Slovaca 44 (1994), 21-34.

[24] MIŠIK, L., Jr.: Sequential completeness and $\{0,1\}$-sequential completeness are different, Czechoslovak Math. J. 34 (1984), 424-431.

[25] NOVÁK, J.: Über die eindeutigen stetigen Erweiterungen stetiger Funktionen, Czechoslovak Math. J. 8 (1958), 344-355.

[26] NOVÁK, J.: On convergence spaces and their sequential envelopes, Czechoslovak Math. J. 15 (1965), 74-100.

[27] NOVÁK, J.: On sequential envelopes defined by means of certain classes of functions, Czechoslovak Math. J. 18 (1968), 450-456.

[28] PAPČO, M.: On measurable spaces and measurable maps, Tatra Mt. Math. Publ. 28 (2004), 125-140.

[29] PAPČO, M.: On fuzzy random variables: examples and generalizations, Tatra Mt. Math. Publ. 30 (2005), 175-185.

[30] PAPČO, M.: On effect algebras, Soft Comput. 12 (2007), 26-35.

[31] PTÁK, P.-PULMANNOVÁ, S.: Orthomodular Structures as Quantum Logics. Fund. Theorie Phys., Vol. 44, Kluwer Acad. Publ., Dordrecht, 1991.

[32] RIEČAN, B.-MUNDICI, D.: Probability on $M V$-algebras, in: Handbook of Measure Theory, Vol. I, II (E. Pap, ed.), North-Holland, Amsterdam, 2002, pp. 869-909.

[33] RIEČAN, B.-NEUBRUNN, T. Integral, Measure, and Ordering. Math. Appl., Vol. 411, Kluwer Acad. Publ., Dordrecht; Ister Science, Bratislava, 1997.

Mathematical Institute

Slovak Academy of Sciences

Grešákova 6

SK-040-01 Košice

SLOVAKIA

E-mail: fric@saske.sk 